\title{
Enhanced distributed hybrid sensor based on Brillouin and Raman scattering combining Fabry-Perot lasers and optical pulse coding
}

\author{
Marcelo A. Soto, Gabriele Bolognini, Fabrizio Di Pasquale \\ Scuola Superiore Sant'Anna, via G. Moruzzi 1, 56124 Pisa, Italy.e-mail: gabriele.bolognini@sssup.it
}

\begin{abstract}
Pulse coding techniques are proposed in combination with the use of Fabry-Perot lasers for simultaneous strain and temperature sensing based on hybrid spontaneous Raman-Brillouin scattering. Combination of both techniques allows for $\sim 0.27 \mathrm{~K} / \sim 30 \mu \varepsilon$ resolution along 25-km sensing range. (C)2010 Optical Society of America

OCIS codes: (060. 2370) Fiber optics sensors, (290.5830) Scattering, Brillouin, (290.5860) Scattering, Raman.
\end{abstract}

\section{Introduction}

Most distributed optical fiber sensors enabling simultaneous strain and temperature measurements are either based on Brillouin scattering [1] or on hybrid Raman-Brillouin scattering effects [2], typically in conjunction with optical time domain reflectometry (OTDR). In hybrid sensors based on Raman-Brillouin scattering [2], the temperature profile along the sensing fiber is directly obtained by measuring the spontaneous anti-Stokes Raman signal, which is strain independent. The temperature profile is then used to estimate the fiber strain derived from Brillouin frequency shift (BFS) measurement (which exhibits a temperature-strain cross-sensitivity). The difference in cross-sections for Brillouin and Raman scattering leads to significantly different backscattered power levels, causing significant inaccuracy issues. The limiting factor in temperature-strain measurements with hybrid sensing is actually related to the noise in the spontaneous Raman scattering (SpRS) measurements [2]. Since it is not possible to increase the input pump power above a given threshold, due to the onset on nonlinear effects, we have recently proposed an effective sensing scheme based on the use of multiple longitudinal modes lasers [3], which allows for higher usable pump power levels as well as low coherent Rayleigh noise (CRN) [4].

In this paper, we propose the use of standard FP lasers combined with optical pulse coding in hybrid RamanBrillouin distributed sensors, for simultaneous strain and temperature measurements. Considering that the typical peak power of each FP mode is far below the nonlinear threshold level, it is possible to use pulse coding techniques [5] to improve the signal-to-noise ratio (SNR) in both acquired Raman and Brillouin traces, leading to significant performance enhancement in hybrid sensors. Combining the use of FP lasers and optical pulse coding techniques, we can achieve a significant SNR enhancement, without exceeding the nonlinear threshold levels, attaining a temperature / strain resolution better than $0.27^{\circ} \mathrm{C} / 30 \mu \varepsilon$ along $25-\mathrm{km}$ sensing range.

\section{Theory}

In distributed hybrid Raman-Brillouin sensors, the anti-Stokes SpRS power and the BFS are simultaneously measured [2]. Since, the anti-Stokes SpRS signal is temperature dependent only, it allows to obtain strainindependent temperature measurements along an optical fiber. In order to get rid of additional losses along the fiber, the ratio of the anti-Stokes Raman power $\left(P_{A S}\right)$ over the Rayleigh-backscattering power $\left(P_{\text {Ray }}\right)$, is typically used, obtaining a dependency on the absolute temperature $(T)$ described by [3]:

$$
\frac{P_{A S}}{P_{\text {Ray }}} \propto\left[\exp \left(\frac{h \Delta v_{R}}{k T}\right)-1\right]^{-1}
$$

where $h$ is the Planck constant, $k$ is the Boltzmann constant, and $\Delta v_{R}$ is the frequency separation between antiStokes Raman and pump light. With the knowledge of the temperature variations $(\Delta T)$ along the sensing fiber, the BFS measurement $\left(\Delta v_{B}\right)$ can be calibrated to obtain the distributed strain $(\Delta \varepsilon)$ according to:

$$
\Delta \varepsilon(z)=\frac{\Delta v_{B}(z)-C_{v_{B} T} \cdot \Delta T(z)}{C_{v_{B} \varepsilon}}
$$

where $C_{v B \varepsilon}=0.048 \mathrm{MHz} / \mu \varepsilon$ and $C_{v B T}=1.07 \mathrm{MHz} /{ }^{\circ} \mathrm{C}$ are the strain and temperature coefficients for BFS [1].

We have recently proposed the use of multi-wavelength sources, such as FP lasers, to improve the performance of distributed hybrid sensors [3], where the main factor limiting is the noise in the anti-Stokes Raman power measurement [2,3]. When FP lasers are used, the peak power launched into the fiber is actually distributed among the different longitudinal modes of the spectrum, overcoming the main limiting nonlinearities, such as stimulated Brillouin scattering (SBS) and modulation instability (MI), particularly relevant when narrowband sources are used. This kind of broadband source allows for the use of high peak power, offering higher SNR in anti-Stokes Raman power measurements, and leading then to a better 
temperature and strain resolutions [3]. On the other hand, the use of optical pulse coding technique has been recently proposed for distributed sensing based on spontaneous Brillouin scattering [5], offering an effective mechanism to improve the SNR of the measurements, and hence to enhance the accuracy of the sensor. However, coding techniques unfortunately also reduce the SBS threshold, so that for instance when using 127bit Simplex coding with relative long pulses, the SBS threshold is decreased down to $10 \mathrm{dBm}$ peak power [5]. Taking into account that the SBS threshold only affects the peak power per mode of the FP laser, the use of pulse coding is still possible when the power of each FP mode remains below the SBS threshold (which is the typical condition even when using high-power FP lasers). These features allow us to combine the advantages of both FP lasers and optical pulse coding in hybrid sensing. We show that the combination of both techniques allows us to significantly improve the SNR of the anti-Stokes Raman power measurement and BFS measurements, leading to a better temperature and strain resolution.

\section{Experimental setup}

Fig. 1 shows the experimental setup used to perform simultaneous strain and temperature measurements based on hybrid RamanBrillouin sensing. Two optical sources, a FP laser and a DFB laser, have been used to compare the performance of the sensor. Since the FP laser allows for more optical power at the fiber input than the DFB laser, an Erbium-doped fiber amplifier (EDFA) has been used to amplify the DFB laser allowing for a better comparison under the same experimental conditions. Note that when using the DFB laser, current dithering

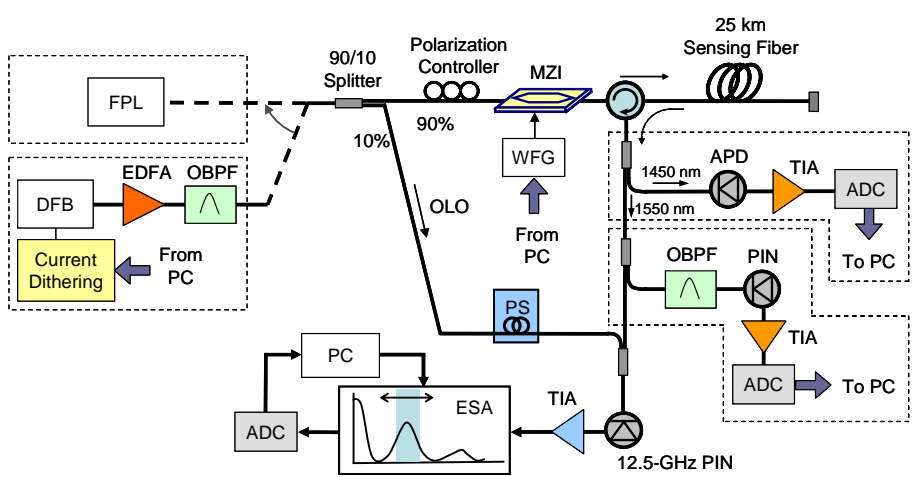

Fig. 1. Experimental set-up of hybrid Raman-Brillouin distributed strain and temperature sensor and wavelength averaging are also required to reduce CRN in the Rayleigh measurements [4]. On the other hand, the broadband characteristic of the FP laser allows us to inherently reduce CRN, not requiring those additional techniques [3]. The CW-light of the source is split into two branches, so that $10 \%$ is used at the receiver side as optical local oscillator (OLO), while the $90 \%$ of the light is modulated by a Mach-Zehnder interferometer (MZI) with 350 ns pulses, allowing for $35 \mathrm{~m}$ spatial resolution. The pulse power at the fiber input is $15 \mathrm{dBm}$ in both cases; which makes the use of coding impossible when employing the DFB laser, due to the detrimental SBS effect taking place at the near fiber-end [5]. On the other hand, considering that the peak power per mode in fiber is $\sim 0 \mathrm{dBm}$ when using the FP laser, which is well below the SBS threshold $(\sim 10 \mathrm{dBm})$ even using coding [5], 127-bit Simplex coding can be effectively employed for further performance enhancement. The sensing fiber is a $25-\mathrm{km}$ standard single-mode fiber, and the receiver-side is composed of three different stages. The first two stages are direct-detection receivers for anti-Stokes Raman and Rayleigh power measurements, making use of an optical band-pass filter (OBPF), trans-impedance amplifiers (TIA) and analogto-digital converters (ADC); the third stage consists in optical and electrical heterodyne receivers for BFS measurements. A polarization scrambler (PS) is used to depolarize the OLO and reduce polarization-induced fluctuations in the optical heterodyne process. The electrical coherent detection is performed by an optical spectrum analyzer (OSA) operating in zero-span mode, with a resolution bandwidth of $3 \mathrm{MHz}$, which actually limits the spatial resolution to $\sim 35 \mathrm{~m}$, however this is not a fundamental limitation to the proposed technique.

\section{Results}

In order to perform distributed strain and temperature measurements based on hybrid Raman-Brillouin sensor using a multi-wavelength optical source, we have employed in our experiment a FP laser with 30 longitudinal modes. Since the peak power per mode is limited by the nonlinear threshold to $10 \mathrm{dBm}$ when using 127-bit Simplex codes, in principle we can use up to $\sim 25 \mathrm{dBm}$ total power at the fiber input without incurring in SBS. In our experiment, the maximum power allowed at the MZI input limits the usable peak power at the fiber input to
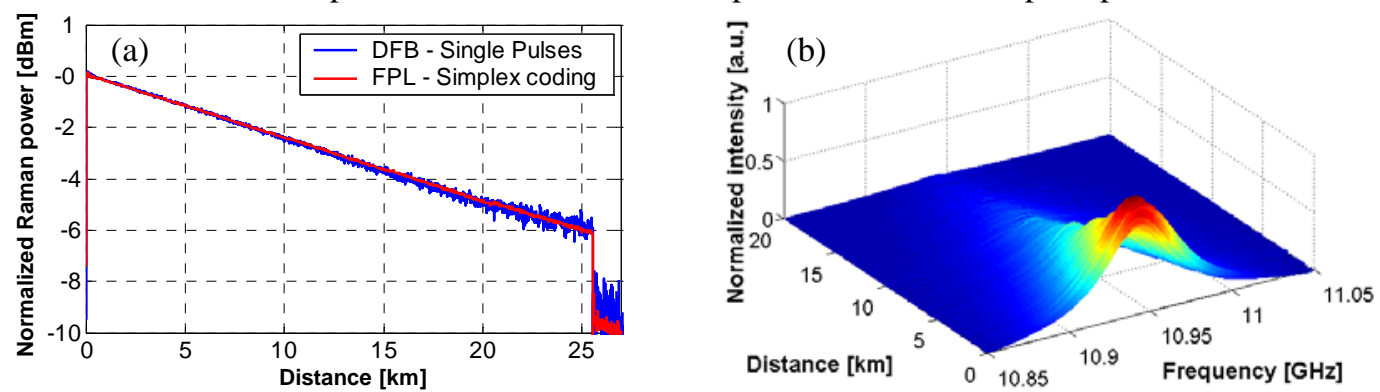

Fig. 2. (a) Anti-Stokes Raman measurements for both DFB laser and FP laser. (b) Brillouin spectrum as a function of the distance when using the FP laser and 127-bit Simplex-codes. 


\section{OThA1.pdf}
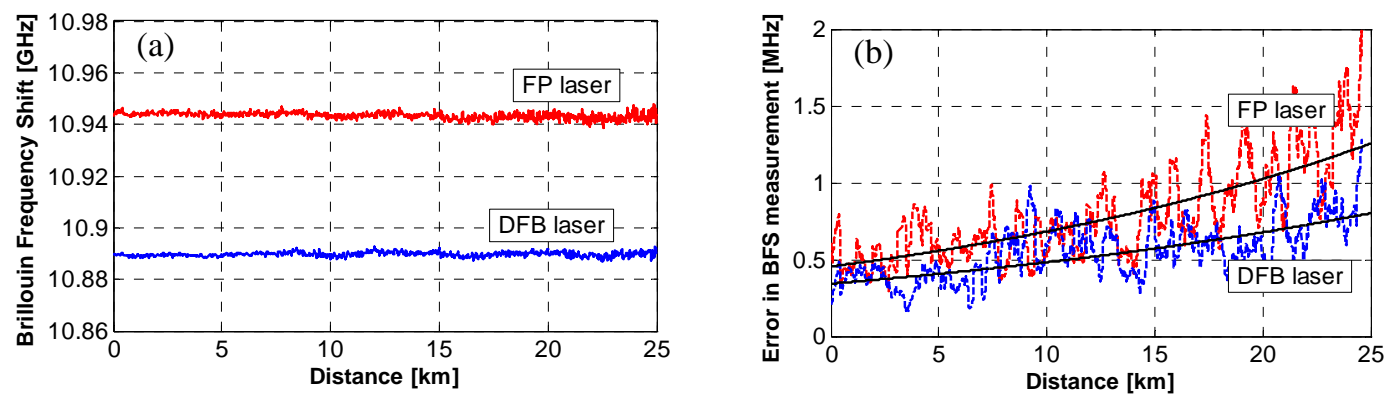

Fig. 3. (a) BFS and, (b) error in the BFS measurement vs distance, for both DFB laser and FP laser.

$15 \mathrm{dBm}$ only. However, this power level is high enough to demonstrate the feasibility of using FP laser combined with Simplex coding with a total power which is higher than the SBS threshold in narrowband sources. Fig. 2a compares the anti-Stokes Raman traces that have been measured considering the same number of acquisitions, i.e. same measurement time, and same input power, for both DFB laser and FP laser with pulse coding. We can clearly observe a much better SNR when using FP laser and coding; we should moreover note that the use of coding with FP laser is possible thanks to its distribution of power among the different longitudinal modes of the spectrum. Actually, if optical pulse codes were applied using the DFB laser, we would obtain a completely distorted trace due to depletion induced by SBS [5]. On the other hand, Fig 2b shows the Brillouin spectrum obtained along the sensing fiber when using Simplex coding, representing the contribution of all Stokes and anti-Stokes light components generated by all FP modes. We can note that, even though the total peak power is higher than SBS threshold with coding and narrowband sources, no distortion of the spectrum is observed, and the spectrum can be in fact properly fitted by a Lorentzian curve in order to obtain the BFS along the fiber. Fig. 3a actually shows the BFS measured in both cases (DFB and FP); a small difference is observed mainly due to the different wavelength of the used DFB and FP lasers. Also note that due to the wavelength dependence of the BFS, each FP mode actually generates a slightly different BFS, inducing an additional small broadening factor in the measured Brillouin spectrum. The measured Brillouin linewidth is actually $\sim 50 \mathrm{MHz}$, reducing the accuracy on the BFS measurement as reported in Fig. 3b. This additional inaccuracy is also partially a consequence of the drift affecting the modes of the FP laser in the $\mu$ sec-scale; this effect is however partiality compensated by the use of Simplex coding. By calculating the standard deviation of both BFS and anti-Stokes Raman power measurements, simultaneous strain and temperature resolution have been calculated. Figs. 4a and $4 \mathrm{~b}$ show a comparison of the experimental temperature and strain resolutions obtained with both DFB laser (using single pulses) and FP laser (using 127-bit Simplex coding). Temperature resolution of $\sim 2{ }^{\circ} \mathrm{C}$, obtained with the DFB laser at $25-\mathrm{km}$ distance, is improved down to a notable value of $\sim 0.27^{\circ} \mathrm{C}$ when using the FP laser and Simplex coding; also the strain resolution is improved from $\sim 60 \mu \varepsilon$ down to $\sim 30 \mu \varepsilon$.
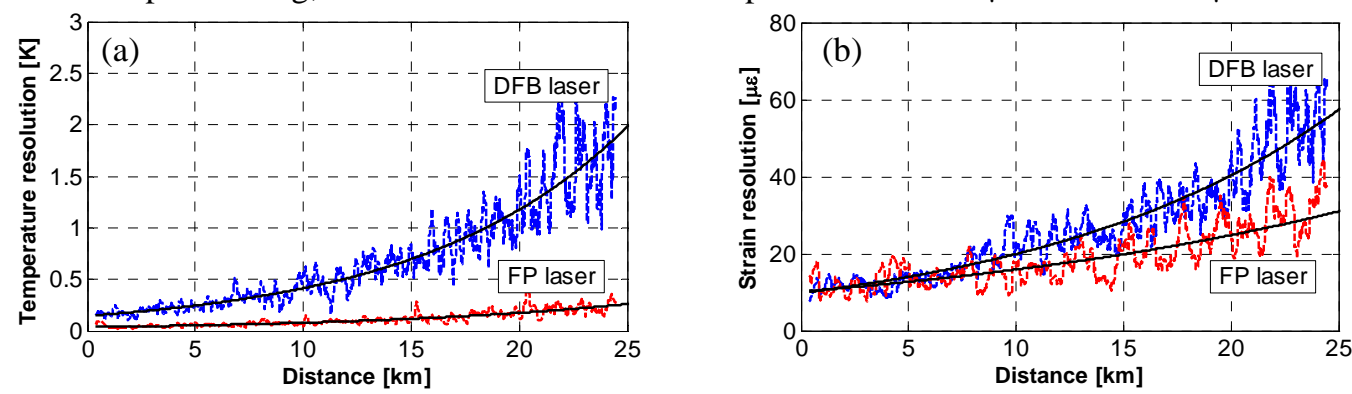

Fig. 4. (a) Temperature and, (b) strain resolution vs distance, for both DFB laser and FP laser.

In conclusion, we have demonstrated the feasibility of combining optical pulse coding techniques with the use of high peak power FP lasers to enhance the performance of hybrid-based sensors. The limitation on the maximum allowed input pulse power when using pulse coding with using narrowband sources can be overcome with FP laser due to the distribution of the power along many different longitudinal modes within the laser spectrum. Thus, multi-wavelength lasers combined with coding techniques provide an effective solution to improve the performance of hybrid sensors, attaining $\sim 0.27^{\circ} \mathrm{C} / \sim 30 \mu \varepsilon$ resolutions at 25 -km distance.

\section{References}

[1] S. M. Maughan et al., "Simultaneous distributed fibre temperature and strain sensor using microwave coherent detection of spontaneous Brillouin backscatter,” Meas. Sci. Technol., vol 12, pp. 834-842, 2001.

[2] M. N. Alahbabi et al., "Simultaneous temperature and strain measurement with combined spontaneous Raman and Brillouin scattering," Opt. Lett., vol. 30, no. 11, pp. 1276-1278, 2005.

[3] G. Bolognini et al., "Fiber-optic distributed sensor based on hybrid Raman and Brillouin scattering employing multi-wavelength FabryPérot lasers,” IEEE Photon. Technol. Lett., vol. 21, no. 20, pp. 1523-1525, 2009.

[4] K. De Souza, "Significance of coherent Rayleigh noise in fibre-optic distributed temperature sensing based on spontaneous Brillouin scattering,” Meas. Sci. Technol., vol. 17, pp. 1065-1069, 2006.

[5] M. A. Soto et al., "Analysis of optical pulse coding in spontaneous Brillouin-based distributed temperature sensors," Opt. Express, vol. 16, n. 23, pp. 19097-19111, 2008. 